\title{
ALEXANDER POLYNOMIALS OF RIBBON LINKS
}

\author{
JONATHAN A. HILLMAN
}

\begin{abstract}
We give a simple argument to show that every polynomial $f(t) \in$ $\mathbb{Z}[t]$ such that $f(1)=1$ is the Alexander polynomial of some ribbon 2-knot whose group is a 1-relator group, and we extend this result to links.
\end{abstract}

It is well known that every Laurent polynomial $f(t) \in \Lambda=\mathbb{Z}\left[t, t^{-1}\right]$ with $f(1)=1$ is the Alexander polynomial of some ribbon 2-knot [7]. (See also [1,2], for the fibred case, and $\S 7 \mathrm{H}$ of [11], for a construction of knot polynomials by surgery.) We shall give another argument, which seems particularly simple, and which gives a slightly stronger result. We shall then extend this result to higher-dimensional links.

In higher dimensions the term "Alexander polynomial" is potentially ambiguous. Let $X=S^{n+2}-i n t K \times D^{2}$ be the knot exterior, $\pi=\pi K=\pi_{1}(X)$ the knot group and $X^{\prime}$ the maximal abelian covering space of $X$. The homology groups $H_{q}\left(X^{\prime} ; \mathbb{Z}\right)$ are finitely generated torsion $\Lambda$-modules under the action of $A u t\left(X^{\prime} / X\right) \cong Z$. They each have a sequence of "Alexander polynomial" invariants $\Delta_{i}^{q}(K)$ such that $\Delta_{i}^{q}(K)$ divides $\Delta_{i+1}^{q}(K)$ in $\Lambda$ [8]. Poincaré duality implies that $\Delta_{i}^{n+1-q}(K)=\overline{\Delta_{i}^{q}(K)}$ for $q \leq\left[\frac{n+1}{2}\right]$, where the overbar is the involution defined by inverting the generators $t_{i}$. More generally, if $L$ is a $\mu$-component $n$-link there are similar invariants in $\Lambda_{\mu}=\mathbb{Z}\left[t_{1}^{ \pm}, \ldots, t_{\mu}^{ \pm}\right]$.

In this paper "Alexander polynomial" shall mean the greatest common divisor $\Delta(\pi)$ of the first nonzero elementary ideal of the "Alexander module" $A(\pi)$ of $\pi$. A presentation for this module may be derived from a presentation for $\pi$ by the free differential calculus. If $n>1$ the module has rank $\mu$ and $\Delta(L)=\Delta_{\mu}^{1}(L)$, but when $n=1$ it has rank $\leq \mu$, with equality if $L$ is concordant to a boundary link. (See [6] for more on Alexander modules.)

Let $\varepsilon: \Lambda_{\mu} \rightarrow \mathbb{Z}$ be the augmentation homomorphism defined by $\varepsilon\left(t_{i}\right)=1$ for all $i$. Then $\varepsilon(\Delta(\pi))=1$, since $\pi / \pi^{\prime} \cong Z^{\mu}$. The burden of this note is that this is the only constraint on such link polynomials, if $n>1$. The case $n=2$ is of particular interest, for then $H_{1}\left(X^{\prime} ; \mathbb{Z}\right)$ and duality determine the other homology modules. (When $n=1$ and $L$ is a boundary link we must also have $\bar{\Delta}=\Delta$; there is as yet no such characterization for other classical links.)

\section{KNOTS}

An $n$-knot is a ribbon knot of 1-fusion if it bounds the sum of two disjoint copies of $D^{n+1}$ along a band $D^{n} \times[0,1]$ which meets the discs only at its ends.

Theorem 1. Let $f=f(t) \in \Lambda$ be such that $f(1)=1$, and let $n>1$. Then there is an $n$-knot $K$ which is a ribbon knot of 1 -fusion such that $\pi^{\prime} / \pi^{\prime \prime} \cong \Lambda /(f)$, where $\pi=\pi K$.

1991 Mathematics Subject Classification. 57Q45.

Key words and phrases. Alexander polynomial. boundary link. knot. 1-relator group. ribbon. 
Proof. We may assume that $f \in \mathbb{Z}[t]$ and $f(0) \neq 0$. Let $d$ be the degree of $f$ and let $g=g(t)=(f(t)-1) /(t-1)$. Then $g(t)=\Sigma_{i=0}^{i=d-1} g_{i} t^{i} \in \mathbb{Z}[t]$. Let

$$
\pi=\left\langle a, t \mid a=w t w^{-1} t^{-1}\right\rangle,
$$

where $w=w(a, t)=\Pi_{i=0}^{i=d-1} t^{i} a^{g_{i}} t^{-i}=a^{g_{0}} \ldots t^{d-1} a^{g_{d-1}} t^{1-d} t^{-g(1)}$. (The final term ensures that $w(1, t)=1$ in $\langle t\rangle$.) Clearly $\pi / \pi^{\prime} \cong Z$, and it is easily seen that $\pi^{\prime} / \pi^{\prime \prime} \cong \Lambda /(f)$, since $f=(t-1) g+1$.

Let $x=a t$ and let $w_{k} \ldots w_{1}$ be a word of length $k$ in the alphabet $\left\{t, t^{-1}, x, x^{-1}\right\}$ representing $w\left(x t^{-1}, t\right)$ Then $\pi$ also has the deficiency-1 Wirtinger presentation

$$
\left\langle t, x_{0}, \ldots, x_{k}, x \mid t=x_{0}, x=x_{k}, x_{i}=w_{i} x_{i-1} w_{i}^{-1} \forall 1 \leq i \leq k\right\rangle .
$$

We may then use the elementary construction of $\S 1.8$ of [6] to obtain an $n$-ribbon $R: D^{n+1} \rightarrow S^{n+2}$ with $\pi$ as its ribbon group, for any $n \geq 1$. This has $k$ parallel throughcuts, and the corresponding slits are in the two extreme components of the complement of the throughcuts. Hence $K=\left.R\right|_{\partial D^{n+1}}$ is the fusion of a 2-component trivial link along a single band, and so is a ribbon knot of 1 -fusion. If $n \geq 2$ then $\pi K \cong \pi$.

The group $\pi$ is a 1-relator group. (This is so for the group of any ribbon knot of 1-fusion.) Since $\pi / \pi^{\prime} \cong Z$ the relator $a t w t^{-1} w^{-1}$ is not a proper power. Therefore c.d. $\pi \leq 2$ [9]. The conditions $c . d . \pi=1, \pi \cong Z$ and $f(t)=1$ are clearly equivalent for groups with such presentations.

When $n=1$ the knot $K=\left.R\right|_{\partial D^{2}}$ provided by the construction of $\S 1.8$ of [6] bounds a disc knot $D^{2} \subset D^{4}$ with group $\pi$, obtained by desingularizing the ribbon immersion $R$, and so $K$ has Alexander polynomial $f \bar{f}$. Ribbon knots realizing such polynomials were first constructed in [12]. In fact, the Alexander polynomial of any classical slice knot has this form [4]. However the ribbon immersion $R$ realizing $\pi$ is not uniquely determined, and we do not know whether we can arrange that $\pi K$ be a 1-relator group.

Addendum. The knot constructed in Theorem 1 is fibred if and only if the extreme coefficients of $f$ are \pm 1 .

Proof. If $f$ is a monic polynomial with $f(0)= \pm 1$ then $\pi^{\prime}$ is free with basis represented by $\left\{t^{i} a t^{-i} \mid 0 \leq i<d\right\}$. Since $n>1$ and $K$ is a ribbon knot of 1 -fusion it is fibred, by a theorem of Yoshikawa $[1,13]$.

The converse is clear.

By taking connected sums of knots we may realize arbitrary finite sequences $\delta_{i}$ with $\delta_{i+1}$ dividing $\delta_{i}$ in $\Lambda$ as the higher polynomial invariants associated to $A(\pi)$. If the summands are all fibred so is their sum.

\section{LINKS}

Let $A=\left\{a_{1}, \ldots, a_{\mu}\right\}$ and $T=\left\{t_{1} \ldots, t_{\mu}\right\}$, and let $\partial_{i}: \mathbb{Z} F(A \cup T) \rightarrow \Lambda_{\mu}$ be the composite of the free derivation $\frac{\partial}{\partial a_{i}}$ of $\mathbb{Z} F(A \cup T)$ with respect to the generator $a_{i}$ with the retraction onto $\mathbb{Z}[F(T)]$ which sends $a_{j}$ to 1 and $t_{j}$ to $t_{j}$ for $j \leq \mu$.

Lemma. Given $f_{i} \in \mathbb{Z}[F(T)]$, there is a word $W \in F(A \cup T)$ with trivial image in $F(T)$ and such that $\partial_{i}(W)=f_{i}$ for all $i \leq \mu$. 
Proof. Suppose that $f_{i}=\Sigma_{m \in F(T)} f_{i m} m$. Let $v_{i}=\Pi_{m \in F(T)} m a_{i}^{f_{i m}} m^{-1}$, where the factors are taken in some fixed order, and let $W=\Pi v_{i}=v_{1} \ldots v_{\mu}$. Then the $v_{i}$ and $W$ have trivial image in $F(T)$. Moreover, $\partial_{i}\left(v_{i}\right)=\Sigma_{m \in F(T)} f_{i m} m=f_{i}$, and $\partial_{j}\left(v_{i}\right)=0$, if $j \neq i$, so $\partial_{i}(W)=\partial_{i}\left(v_{i}\right)=f_{i}$, for all $i \leq \mu$.

The order on $F(T)$ used in this lemma is not important.

Theorem 2. Let $f \in \Lambda_{\mu}$ be such that $\varepsilon(f)=1$, and let $n>1$. Then there is a $\mu$-component ribbon boundary $n$-link $L$ with $\Delta(\pi L)=f$.

Proof. We may write $f=1-\Sigma\left(t_{i}-1\right) f_{i}$. Choose $F_{i} \in \mathbb{Z}[F(T)]$ with image $f_{i} \in \Lambda_{\mu}$. There are words $W_{i} \in F(A \cup T)$ with trivial image in $F(T)$ and such that $\partial_{j} W_{i}=F_{i}$ for all $j \leq \mu$, by the lemma. Let $\pi$ be the group with presentation

$$
\left\langle a_{i}, t_{i}, 1 \leq i \leq \mu \mid a_{i}=t_{i} W_{i} t_{i}^{-1} W_{i}^{-1}, \forall i\right\rangle .
$$

The free differential calculus gives a presentation matrix $\left[I_{\mu}-D, 0_{\mu}\right]$ for $A(\pi)$, where $D$ is a $\mu \times \mu$ matrix with $D_{i j}=\left(t_{i}-1\right) f_{i}$ for all $i, j \leq \mu$ and $0_{\mu}$ is a null $\mu \times \mu$ matrix. As the columns of $D$ are all equal, it is easy to see that $\Delta(\pi)=$ $\operatorname{det}\left(I_{\mu}-D\right)=1-\Sigma\left(t_{i}-1\right) f_{i}=f$.

As in Theorem 1, the group $\pi$ has an equivalent Wirtinger presentation of deficiency $\mu$, and the elementary construction of $\S 1.8$ of [6] gives a $\mu$-component ribbon $n$-link $L$ with group $\pi L \cong \pi$ and meridians corresponding to the generators $t_{i}$. Since the projection of $\pi$ onto $\pi /\left\langle\left\langle a_{1}, \ldots, a_{\mu}\right\rangle\right\rangle \cong F(T)$ carries the meridians to a free basis, $L$ is a boundary link.

Let $Y$ be the finite 2-complex corresponding to the above presentation, and let $Z$ be the complex obtained by adjoining 2-cells along maps corresponding to the generators $t_{i}$. Then $Z$ is 1 -connected and $\chi(Z)=1$, and so it is a finite contractible 2-complex. Thus if the Whitehead Conjecture is true $Y$ is aspherical, and so $c . d . \pi L \leq 2$.

When $n=1$ this construction gives a ribbon boundary link $L$ with $\Delta(\pi)=$ $f \bar{f}$. This condition is satisfied by the first nonzero Alexander polynomial of every classical slice link. Every $f \in \Lambda_{\mu}$ such that $\varepsilon f=1$ and $\bar{f}=f$ is $\Delta(\pi)$ for some $\mu$-component boundary 1-link [5].

\section{A NON-COMMUtATive ANAlogue?}

A $\mu$-component link $L$ is an homology boundary link if there is an epimorphism from $\pi=\pi L$ to $F(\mu)$. (It is a boundary link if and only if there is such an epimorphism which takes the images of a set of meridians to a basis for the free group.) The kernel of any such epimorphism is $\pi_{\omega}=\cap \pi_{[n]}$, the intersection of the lower central series. Let $k$ be a field and $k \Gamma_{\mu}=k[F(\mu)]$. The homology groups $H_{q}=H_{q}\left(X^{\omega} ; k\right)$ of the covering space $X^{\omega}$ with group $\pi_{\omega}$ are finitely generated left $k \Gamma_{\mu}$-modules. If $1 \leq q<n$ then $H_{q}$ satisfies the Sato property: $k \otimes_{k \Gamma_{\mu}} H_{q}=$ $\operatorname{Tor}_{1}^{k \Gamma_{\mu}}\left(k, H_{q}\right)=0$. (See Chapter 9 of $[6]$.)

Farber constructed invariants of such modules with values "noncommutative rational functions". When $\mu=1$ these are equivalent to the usual Alexander polynomials $\Delta_{0}^{q}$ (although closer in form to the logarithmic derivative) [3]. His work has been reformulated in terms of Gelfand-Retakh quasideterminants [10]. Is there a realization result analogous to Theorem 2 for the invariants of $H_{1}\left(X^{\omega} ; k\right)=$ $k \otimes_{\mathbb{Z}}\left(\pi_{\omega} / \pi_{\omega}{ }^{\prime}\right) ?$ 


\section{REFERENCES}

[1] Aitchison, I.R. and Silver, D.S. On certain fibred ribbon disc pairs, Trans. Amer. Math. Soc. 306 (1988), 529-551.

[2] Asano, K. and Yoshikawa, K. On polynomial invariants of fibered 2-knots, Pacific J. Math. 97 (1981), 267-269.

[3] Farber, M.S. Noncommutative rational functions and boundary links, Math. Ann. 293 (1992), 543-568.

[4] Fox, R.H. and Milnor, J.W. Singularities of 2-spheres in 4-space and cobordism of knots, Osaka J. Math. 3 (1966), 257-267.

[5] Friedl, S. Algorithm for finding boundary link Seifert matrices, J. Knot Th. Ramif. 15 (2006), 601-612.

[6] Hillman, J.A. Algebraic Invariants of Links, Series on Knots and Everything, vol. 32, World Scientific Publishing Co. (2002).

[7] Kinoshita, S. On the Alexander polynomials of 2-spheres in a 4-sphere, Ann. Math. 74 (1961), 518-531.

[8] Levine, J. Polynomial invariants of knots in codimension two, Ann. Math. 84 (1966), 537-554.

[9] Lyndon, R. C. Cohomology theory of groups with a single defining relation. Ann. of Math. 52, (1950). 650-665.

[10] Retakh, V., Reutenauer, C. and Vaintrob, A. Noncommutative rational functions and Farber's invariants of boundary links, in Differential topology, infinite-dimensional Lie algebras, and applications, Amer. Math. Soc. Transl. Ser. 2, 194, Amer. Math. Soc., Providence, RI (1999), 237-246.

[11] Rolfsen, D. Knots and Links, Publish or Perish, Inc., Berkeley, Cal. (1976).

[12] Terasaka, H. On null-equivalent knots, Osaka J. Math. 11 (1959), 95-113.

[13] Yoshikawa, K. On fibering a class of $n$-knots, Math. Sem. Notes Kobe Univ. 9 (1981), 241-245.

School of Mathematics and Statistics F07, University of Sydney, NSW 2006, AusTRALIA

E-mail address: jonh@maths.usyd.edu.au 